\title{
The Numerical Implementation of the Linear Integro- Differential Equations Method for the Solution of Non-Stationary Heat Conduction Problem with Two Space Variables
}

\author{
I. V. Sergienko ${ }^{1}$, O. N. Lytvyn², O. O. Lytvyn², V. I. Mezhuyev ${ }^{3 *}$, L. S. Lobanova² and G. V. Zalyzhna² \\ ${ }^{1}$ Institute of Cybernetics of the National Academy of Science, Kyiv, Ukraine \\ 2Ukrainian Engineering and Pedagogical Academy, Kharkiv, Ukraine \\ 3 University Malaysia Pahang, Gambang, Malaysia \\ mejuev@ukr.net ${ }^{3}$
}

\begin{abstract}
The paper proposes a general method for solution of non-stationary heat conduction problem with two space variables. It is based on Finite Element Method, which uses the operator of the interlination on a given system of lines. The region of consideration divided on rectangular or triangular elements with curvilinear sides belonging to the boundary of the domain. Interlineation of functions is a restoration of the function with the help of its traces on the system of lines. Operators of interlineations allow to restore functions of two or more variables between the lines using their traces and traces of their partial derivatives up to a given order on the indicated lines. Application of the method on examples of solutions of the nonstationary heat conduction equation for regions of complex shape is illustrated.
\end{abstract}

Keywords: Interlination of Functions, Initial-Boundary Value Problem, Linear Integro-Differential Equations, I NonStationary Heat Conduction Equation

\section{Introduction}

There are number of works devoted to the numerical implementation of the Linear Integro-Differential Equations (LIDE) method for the non-stationary heat conduction equation with two space variables (see e.g. ${ }^{1-4}$ ). In the ${ }^{5-8}$ we proposed the method LIDE on the base on interlineation of functions?

Operators of interlineation allow us to restore functions of two or more variables between the intended lines using their traces and traces of their partial derivatives up to a given order on indicated lines. They have a very high precision. The same large accuracy also retains for approximated solutions, found by the LIDE method.
In $^{1-5}$ the finite element method for solving initialboundary value problem was studied for non-stationary heat equation

$$
\frac{\partial u}{\partial t}-\frac{\partial}{\partial x}\left(p_{1}(x, y) \frac{\partial u}{\partial x}\right)-\frac{\partial}{\partial y}\left(p_{2}(x, y) \frac{\partial u}{\partial y}\right)+q(x, y) u=f(x, y, t)
$$

with two space variables using formulas of spline interpolation, based on spline interlination of functions $u(x, y, t)$ by the spatial variables $x, y$.

The method gives an approximate solution in the form of formulas of spline interpolation for spatial variables with coefficients, that are functions of the variable $t$. The method is based on replacing traces by approximate solution (splines) in the interlination formulas used for their

${ }^{*}$ Author for correspondence 
presentation, and is a finite cell method implementation of LIDE method for solving partial differential equations (method of reduction to a system of Linear Integro-Differential Equations - LIDE). In particular, in the papers ${ }^{4-7}$ we have considered the use of this method for solving non-stationary heat conduction problem for the case of the areas, which are defined as the union of rectangles with sides parallel to the axes.

In this paper, this method is applied to the regions bounded by arcs of known curves.

\section{Materials and Methods}

Let's consider the general method for constructing the function $u(x, y, t)$ that exactly satisfies the Dirichlet boundary conditions of arbitrary domain $G \subset R^{2}$. To develop the structure of solutions of initial boundary value problems, we use the $\operatorname{method}^{3}$ for constructing the functions of two variables $x, y$ that exactly satisfy the boundary conditions on the boundary of two-dimensional domains having complex shapes.

From practical point of view, it is important to build interlination operators on lines for rectangulation and triangulation in the areas of complex shape, bounded by arcs of known curves. We suppose that $G \subset R^{2}$ is a bounded domain on the plane, the boundary $\partial \mathrm{G}$ of which is a union of arcs of known curves.

Let's assume, without loss of generality, that the area $G$ is completely placed in a rectangle $[a, b] \times[c, d]$. We divide $G$ into subregions by the lines $x=x_{k} k=\overline{0, M_{1}}$ and $y=y_{l}$ $l=\overline{0, M_{2}}, a=x_{0}<x_{1}<\ldots<x_{M_{1}}=\mathrm{b} ; c=y_{0}<y_{1}<\ldots<y_{M_{2}}=\mathrm{d}$.

These subregions can be the rectangles $R_{i, j}=\left[x_{i}, x_{i+1}\right]$ $\times\left[y_{j}, y_{j+1}\right] \subset G$ or the quadrangles $R_{i, j}^{(1)}=\left[x_{i}, x_{i+1}(y)\right] \times\left[y_{j}\right.$, $\left.y_{j+1}\right] \subset G, R_{i, j}^{(2)}=\left[x_{i}, x_{i+1}(y)\right] \times\left[y_{j}, y_{j+1}(x)\right] \subset G, R_{i, j}^{(3)}=\left[x_{i}(y)\right.$, $\left.x_{i+1}\right] \times\left[y_{j}, y_{j+1}\right] \subset G, R_{i, j}^{(4)}=\left[x_{i}, x_{i+1}\right] \times\left[y_{j}(x), y_{j+1}\right] \subset G$ in which three sides are parallel to the axes, and one is, generally speaking, curved side (a part of the border region $\partial \mathrm{G})$. In addition, these subregions can be triangles

$$
\begin{aligned}
& T_{i, j}^{(1)}=\left\{(x, y) \mid x_{i} \leq x \leq x_{i+1}, y_{j} \leq y \leq \eta_{j+1}(x), \eta_{j+1}^{\prime}(x)<0\right\}, \\
& T_{i, j}^{(2)}=\left\{(x, y) \mid x_{i-1} \leq x \leq x_{i}, y_{j} \leq y \leq \eta_{j+1}(x), \eta_{j+1}^{\prime}(x)>0\right\}, \\
& T_{i, j}^{(3)}=\left\{(x, y) \mid x_{i-1} \leq x \leq x_{i}, \eta_{j-1}(x) \leq y \leq y_{j}, \eta_{j-1}^{\prime}(x)<0\right\}, \\
& T_{i, j}^{(4)}=\left\{(x, y) \mid x_{i} \leq x \leq x_{i+1}, \eta_{j-1}(x) \leq y \leq y_{j}, \eta_{j-1}^{\prime}(x)>0\right\},
\end{aligned}
$$
$\partial \mathrm{G}$.

in which one curved side is a part of the border region
We write the formula for interlination on quadrangle $R_{i, j}^{(1)}=\left[x_{i}, x_{i+1}\right] \times\left[y_{j}, y_{j+1}(x)\right] \subset G$, in which one side is curved part of the border region $\partial \mathrm{G}$ :

$$
\begin{aligned}
& O_{i, j}^{(1)} F(x, y, t)=\left(P_{1}+P_{2}-P_{1} P_{2}\right) F(x, y, t), \\
& P_{1} F(x, y, t)=\frac{y-y_{j+1}(x)}{y_{j}-y_{j+1}(x)} F\left(x, y_{j}, t\right) \frac{y-y_{j}}{y_{j+1}(x)-y_{j}} F\left(x, y_{j+1}(x), t\right), \\
& P_{2} F(x, y, t)=\frac{x-x_{i+1}}{x_{i}-x_{i+1}} F\left(x_{i}, y, t\right) \frac{x-x_{i}}{x_{i+1}-x_{i}} F\left(x_{i+1}, y, t\right), \\
& P_{1} P_{2} F(x, y, t)=\frac{y-y_{j+1}(x)}{y_{j}-y_{j+1}(x)} P_{2} F\left(x, y_{j}, t\right) \frac{y-y_{j}}{y_{j+1}(x)-y_{j}} P_{2} F\left(x, y_{j+1}(x), t\right) .
\end{aligned}
$$

Theorem 1. Operator $O_{i, j}^{(1)} F(x, y, t)$ interlinates the function $F(x, y, t) \in C\left(R_{i, j}^{(1)}\right)$ on the boundary of the quadrilateral $R_{i, j}^{(1)}$ with curved side: $O_{i, j}^{(1)} F(x, y, t)=F(x$, $y, t),(x, y) \in \partial R_{i, j}^{(1)}$ that is $O_{i, j}^{(1)} F\left(x_{q}, y, t\right)=F\left(x_{q}, y, t\right), q=i$, $i+1 ; O_{i, j}^{(1)} F\left(x, y_{j}, t\right)=F(x, y, t), O_{i, j}^{(1)} F\left(x, y_{j+1},(x), t\right)=F$ $\left(x, y_{j+1}(x), t\right)$.

The proof is provided by the direct examination.

Similarly, interlinants are built for interlination of function $F(x, y, t)$ on the sides of quadrilaterals $R_{i, j}^{(2)}, R_{i, j}^{(3)}$, $R_{i, j}^{(4)}$ with curved sides.

Now let us write the formula for interlination on a right-angled triangle with curved hypotenuse (that the triangle with sides parallel to the axes and curved hypotenuse). We consider the right triangle with vertices $\mathrm{O}(0,0)$, $\mathrm{A}(1,0), \mathrm{B}(0,1)$ and the hypotenuse $A \breve{B}$, which is defined by the equation $f(x)+g(y)=1$, where functions $f(x), g(y)$ are continuous and monotone on $[0,1]$ and satisfy the conditions $f(0)=0, f(1)=1, g(0)=0, g(1)=1$.

Theorem 2. Let $F(x, y, t) \in C(T), P_{1} F(x, y, t)=f(x)$ $F\left(f^{-1}(1-g(y)), y, t\right)+g(y) F\left(x, g^{-1}(1-f(x)), t\right), P_{2} F(x, y, t)$ $=F(x, 0, t)+F(0, y, t)-F(0,0, t)$.

Then the operator

$P_{12} F(x, y, t)=\left(P_{1} \oplus P_{2}\right) F(x, y, t):=\left(P_{1}+P_{2}-P_{1} P_{2}\right) F(x, y$, $t)=f(x) F\left(f^{-1}(1-g(y)), y, t\right)+g(y) F\left(x, g^{-1}(1-f(x)), t\right)+$ $F(x, 0, t)+F(0, y, t)-\mathrm{F}(0,0, t)-f(x)\left[F(0, y, t)+F\left(f^{-1}(1-\right.\right.$ $g(y), 0, t)-F(0,0, t)]-g(y)[F(0, g-1(1-f(x)), t)+F(x, 0$, $t)-F(0,0, t)]$

interlinates the function $F(x, y, t)$ on three sides of the triangle $T_{i, j}$, that is

$$
\begin{gathered}
P_{12} F(x, 0, t)=F(x, 0, t), P_{12} F(0, y, t)=F(0, y, t), \\
P_{12} F(x, y, t)=F(x, y, t), \text { if } f(x)+g(y)=1
\end{gathered}
$$

Proof. We write formula (1) in the form

$P_{12} F(x, y, t)=(1-f(x)-g(y))[F(x, 0, t)+F(0, y, t)-F(0$, $0, t)]+f(x)\left[F\left(f^{-1}(1-g(y)), y, t\right)-F\left(f^{-1}(1-g(y)), 0, t\right)+\right.$ $F(x, 0, t)+g(y)\left[F\left(x, g^{-1}(-f(x)), \mathrm{t}\right)-F\left(0, g^{-1}(1-f(x)), t\right)\right.$ $+F(0, y, t)]$ 
If we put in the formula (4) $y=0$, we have :

$P_{12} F(x, 0, t)=(1-f(x)) F(x, 0, t)+f(x)\left[F\left(f^{-1}(1), 0, t\right)-\right.$ $F\left(f^{-1}(1), 0, t\right)+F(x, 0, t)=F(x, 0, t)$

Similarly,

$P_{12} F(0, \mathrm{y}, t)=(1-g(y)) F(0, \mathrm{y}, t)+g(y)\left[F\left(0, g^{-1}(1), t\right)-F(0\right.$, $\left.\left.g^{-1}(1), t\right)+F(0, y t)\right]=F(0, y, t)$

Thus, the ratio (2) is proven.

Substituting $y=g^{-1}(1-f(x))$ in the formula (4), we obtain:

$$
\begin{aligned}
& P_{12} F\left(x, g^{-1}(1-(x)), t\right)= \\
& =f(x)\left[\begin{array}{l}
F\left(f^{1}\left(1-g\left(g^{-1}(1-f(x))\right)\right), g^{-1}(1-f(x)), t\right) \\
-F\left(f^{-1}\left(1-g\left(g^{-1}(1-f(x))\right)\right), 0, t\right)+F(x, 0, t)
\end{array}\right]+ \\
& +g\left(g^{-1}(1-f(x))\right)\left[\begin{array}{l}
F\left(x, g^{-1}(1-f(x)), t\right) \\
-F\left(0, g^{-1}(1-f(x)), t\right) \\
+F\left(0, g^{-1}(1-f(x)), t\right)
\end{array}\right]= \\
& =f(x)\left[\begin{array}{l}
F\left(f^{-1}(f(x)), g^{-1}(1-f(x)), t\right) \\
-F\left(f^{-1}(f(x)), 0, t\right)+F(x, 0, t)
\end{array}\right]+ \\
& +(1-f(x))\left[F\left(x, g^{-1}(1-f(x)), t\right)\right]= \\
& =f(x)\left[F\left(x, g^{-1}(1-f(x)), t\right)-F(x, 0, t)+F(x, 0, t)\right]+ \\
& +(1-f(x))\left[F\left(x, g^{-1}(1-f(x)), t\right)\right]=F\left(x, g^{-1}(1-f(x)), t\right) .
\end{aligned}
$$

Similarly, we prove that $P_{12} F\left(f^{-1}(1-g(y)), y, t\right)$ $=F\left(f^{-1}(1-g(y)), y, t\right)$.

Thus, we prove property (3). Theorem 2 is proven.

Theorem 3. Let operators $O R_{i, j}^{(q)} F(x, y, t), q=0,1, \ldots 4$, interlinate the function $F(x, y, t)$ on the sides of quadrilaterals $R_{i, j}^{(q)}, q=0,1, \ldots, 4$, and operators $O T_{i, j}^{(q)}, F(x, y, t), q$ $=1, \ldots, 4$, interlinate the function $F(x, y, t)$ on the sides of triangles $T_{i, j}^{(q)} \subset \mathrm{G}$ with, generally speaking, curved hypotenuse (as was outlined above).

Then the operator

$$
O_{g} F(x, y, t)=\left\{\begin{array}{ll}
O R_{i, j}^{(q)} F(x, y, t), & (x, y) \in R_{i, j}^{(q)}, q=0 \vee 1 \vee \ldots \vee 4 \\
O T_{i, j}^{(q)} F(x, y, t), & (x, y) \in T_{i, j}^{(q)}, q=1 \vee \ldots \vee 4
\end{array}, t \geq \geq\right.
$$

0 , interlinate the function $F(x, y, t)$ on the lines $x=x_{k} \in$ $[a, b], y=y_{i} \in[c, d]$, and on the boundary $\partial G$ of an arbitrary region $G$, that is $O_{G} F\left(x_{k}, y, t\right)=F\left(x_{k}, y, t\right), O_{G} F(x$, $\left.y_{i}, t\right)=F\left(x, y_{i}, t\right), O_{G} F(x, y, t)=F(x, y, t),(x, y) \in \partial G$. At that condition $O_{G} F(x, y, t) \in C(G) \forall F(x, y, t) \in C(G)$ $\forall t \geq 0$. This suggests that $O_{G} F\left(x_{k}, y, t\right) \in W^{1}{ }_{2}(G) \forall t \geq$ 0 , because

$$
\begin{aligned}
& \iint_{G}\left[\left(O_{G} F\right)^{2}+\left(\frac{\partial O_{G} F}{\partial x}\right)^{2}+\left(\frac{\partial O_{G} F}{\partial y}\right)^{2}\right] d x d y \\
& =\sum_{R_{i j}^{(q)} \subset G} \iint_{R_{i i^{(q)}}}\left[\left(O_{G} F\right)^{2}+\left(\frac{\partial O_{G} F}{\partial x}\right)^{2}+\left(\frac{\partial O_{G} F}{\partial y}\right)^{2}\right] d x d y \\
& +\sum_{T_{i j^{(q)}}^{(q)}} \iint_{T_{i j}^{(q)} \subset G}\left[\left(O_{G} F\right)^{2}+\left(\frac{\partial O_{G} F}{\partial x}\right)^{2}+\left(\frac{\partial O_{G} F}{\partial y}\right)^{2}\right] d x d y<\infty .
\end{aligned}
$$

Proof. The fact that $O_{G} F(x, y, t) \in C(\bar{G}) \forall t \geq 0$ implies from the fact that when building operators $O R_{i, j}^{(q)} F(x, y$, $t$ ) and $O T_{i, j}^{(q)} F(x, y, t)$, in each elements $R_{i, j}^{(q)}, T_{i, j}^{(q)}$ operator $\mathrm{O}_{\mathrm{G}} \mathrm{F}$ represented as a formula of interlination, which uses the same tracks on the border between the elements, and keeps continuity with the transition from one element to another. At the same time, corresponding formula of interlination is continuous by construction in each of these elements (assuming that $F \in C(\bar{G})$, get $F\left(x_{k}, y, t\right) \in$ $C(R), F(x, y, t) \in C(R) \forall t \geq 0)$.

The fact that the formula $O_{G} F(x, y, t)$ satisfies the relevant interlination conditions on the lines $x=x_{k}$ and $y=$ $y_{j}$, is derived from the fact that operators $O R_{i, j}^{(q)} F(x, y, t)$ and $O T_{i, j}^{(q)} F(x, y, t)$ by construction on the boundary of its elements have traces which are the traces of functions $F(x, y, t)$, that is $\left.F\left(x_{k}, y, t\right)\right|_{y-y_{i}}=\left.F\left(x, y_{j}, t\right)\right|_{x-x_{k}} \forall t \geq 0$. Theorem 3 is proven.

Remarks. If tracks $F\left(x_{k}, y, t\right)=\varphi_{k}(y, t), F\left(x, y_{i}, t\right)=\psi_{i}$ $(x, t)$ are unavailable, than they can be replaced by some functions (polynomials, splines, etc.) with unknown parameters. As a result, $O_{G} F(x, y, t)$ becomes a function that coincides with the function $F(x, y, t)$ on the boundary $\partial G$ of the region $G$ regardless of the choice of the above parameters. This type of function can be used to approximate functions $F(x, y, t)$, based on certain requirements on the choice of the unknown parameters.

Relatively, following statements are valid for interlination on the triangle.

Theorem 4. Let

$$
\begin{aligned}
& P_{12} F(x, y, t)=(1-f(x)-g(y))[F(x, 0, t)+F(0, y, t)-F(0,0, t)]+ \\
& +f(x)\left[F\left(f^{-1}(1-g(y)), y, t\right)-F\left(f^{-1}(1-g(y)), 0, t\right)+F(x, 0, t)\right]+ \\
& +g(y)\left[F\left(x, g^{-1}(1-f(x)), t-F\left(0, g^{-1}(1-f(x)), t\right)+F(0, y, t)\right]\right.
\end{aligned}
$$

Then for the remainder $R_{12} F(x, y, t)=\left(I-P_{12}\right) F(x, y, t)$ $\forall F \in C^{1,1}(T)$ relation holds: 


$$
\begin{aligned}
& R_{12} F(x, y, t)=(1-f(x)-g(y)) \int_{0}^{x} \int_{0}^{y} F^{(1,1,0)}(\xi, \eta, t) d \xi d \eta \\
& +f(x) \int_{\xi=x}^{f^{-1}(1-g(y))} \int_{\eta=0}^{y} F^{(1,1,0)}(\xi, \eta, t) d \xi d \eta \\
& +g(y) \int_{\xi=0}^{x} \int_{\eta=y}^{g^{-1}(1-f(x))} F^{(1,1,0)}(\xi, \eta, t) d \xi d \eta
\end{aligned}
$$

Proof. Let us write the equations

$$
\begin{aligned}
F(x, y, t) & =F(x, 0, t)+f(0, y, t)-F(0,0, t)+\int_{0}^{x} \int_{0}^{y} F^{(1,1,0)}(\xi, \eta, t) d \xi d \eta \forall t \geq 0, \\
F(x, y, t) & =F\left(f^{-1}(1-g(y)), y, t\right)-F\left(f^{-1}(1-g(y)), 0, t\right)+F(x, 0, t)- \\
& -\int_{\xi=x}^{f^{-1}(1-g(y))} \int_{\eta=0}^{y} F^{(1,1,0)}(\xi, \eta, t) d \xi d \eta \quad \forall t \geq 0, \\
F(x, y, t) & =F\left(x, g^{-1}(1-f(x)), t\right)-F\left(0, g^{-1}(1-f(x)), t\right)+F(0, y, t)- \\
& -\int_{\xi=0}^{x} \int_{\eta=y}^{g^{-1}(1-f(x))} F^{(1,1,0)}(\xi, \eta, t) d \xi d \eta \quad \forall t \geq 0 .
\end{aligned}
$$

With their help we obtain the equality:

$$
F(x, y, t) \equiv(1-f(x)-g(y)) F(x, y, t)+f(x) F(x, y, t)+g(y) F(x, y, t)
$$
$\equiv P_{12} F(x, y, t)+R_{12} F(x, y, t)$ Theorem 4 is proved.

Consequence. The equality is valid:

$P_{12} F(x, y, t)=F(x, y, t) \forall F(x, y, t)=\varphi(x, t)+\psi(y, t) \forall F \geq 0$, there $\varphi(x, t), \psi(y, t) \in C^{1}[0,1] \forall t \geq 0$ are arbitrary function of two variables.

The proof follows from the fact that $\frac{\partial^{2}}{\partial x \partial y}(\varphi(x, t)+\psi(y, t))=0$, so that $R_{12}(\varphi(x, t)+\psi(y, t))=0$; therefore, $P_{12}(\varphi(x, t)+\psi(y, t))=\varphi(x, t)+\psi(y, t) \forall t \geq 0$

\section{Results and Discussion}

To prove applicability of our approach, let's consider two important examples.

Example 1. In the finite elements method for solving boundary value problems for differential equations with partial derivatives, in the case of complex shape regions, satisfaction of the boundary conditions (especially heterogeneous) is very important. Here, it is expedient to use interlination of functions.

Example 2. Applications of the operators for functions interlination are also useful in the method of least squares, when approaching function uses a finite number of its values at interior points of the region $G$ and through its traces on the arcs of known curves within the region $G$, or curves such that their union is the boundary of region
G, etc. It should be emphasized that classical version of the method of least squares approximation of functions of two variables uses only function values at some system of points (or some other system of functionals of the function) and does not use traces of the function on some lines. Obviously, such the information as traces of function on some system of lines are very helpful.

Taking these examples in mind, let's formulate next two statements.

Theorem 5. Let $\mathrm{G}$ is the triangle with curved boundary $\partial G, G=\{x \geq 0, y \geq 0, f(x)+g(y) \leq 1\}$ and $F(x, y, 0)=\varphi(x, y)$. Then the function $H(x, y, t)=\varphi(x, y)+U(x, y, t)-U(x, y, 0)$, there $U(x, y, t)=P_{12} F(x, y, t)$, satisfies the initial $H(x, y, 0)=\varphi(x, y)$ and boundary $H(x, y, t)=U(x, y, t),(x, y) \in \partial G$ conditions $F(x, y, t)$ is arbitrary continuous in $G$ function), that is $\left.P_{12} F(x, y, 0)\right|_{\partial G}=\left.\varphi(x, y)\right|_{\partial G}, \quad P_{12} F(x, y, \mathrm{t})=F(x, y, t) \quad \forall(x, y) \in \partial G$ and $\forall t \geq 0$.

Proof. Indeed, under the assumption,

$$
\begin{gathered}
\varphi(o, y)=U(0, y, 0)=F(0, y, 0), \varphi(x, 0)=U(x, 0,0)=F(x, 0,0), \\
\varphi\left(x, g^{-1}(1-f(x))\right)=U\left(x, g^{-1}(1-f(x)), 0\right)=F\left(x, g^{-1}(1-f(x)), 0\right) . \\
\text { Therefore, } H(x, y, 0)=\varphi(x, y)+U(x, y, 0)-U(x, y, 0)=\varphi(x, y) ; \\
H(0, y, t)=\varphi(0, y)+U(0, y, t)-U(0, y, 0)=U(0, y, t) ; \\
H(x, 0, t)=\varphi(x, 0)+U(x, 0, t)-U(x, 0,0)=U(x, 0, t) ; \\
H\left(x, g^{-1}(1-f(x)), t\right)=\varphi\left(x, g^{-1}(1-f(x))\right)+U\left(x, g^{-1}(1-f(x)), t\right) \\
-U\left(x, g^{-1}(1-f(x)), 0\right)=U\left(x, g^{-1}(1-f(x)), t\right) .
\end{gathered}
$$

Theorem 5 is proven.

Consequence. The function $u(x, y, t)=\varphi(x, y)+U(x, y, t)-$ $U(x, y, 0)$ is an exact solution of the initial-boundary value problem

$$
\begin{gathered}
\frac{\partial u}{\partial t}=\frac{\partial}{\partial x}\left(p(x, y) \frac{\partial u}{\partial x}\right)+\frac{\partial}{\partial y}\left(q(x, y) \frac{\partial u}{\partial y}\right)+f(x, y, t), t>0,(x, y) \in G, \\
u(x, y, 0)=\varphi(x, y),(x, y) \in G, \\
u(x, y, t)=U(x, y, t),(x, y) \in \partial G
\end{gathered}
$$

provided that

$$
\begin{aligned}
& f(x, y, t)=\frac{\partial u}{\partial t}-\frac{\partial}{\partial x}\left(p(x, y) \frac{\partial u}{\partial x}\right)-\frac{\partial}{\partial y}\left(q(x, y) \frac{\partial u}{\partial y}\right) \\
& U(x, y, t)=P_{12} F(x, y, t), F(x, y, t) \in C^{(1,1,0)}(G)
\end{aligned}
$$

The proof is obtained using Theorem 5 and direct substitution $F(x, y, t)$ in equation (6) and taking into account equation (7).

Example. Consider the problem of non-stationary heat conduction in region $G$, which is an equilateral trapezoid 


$$
\begin{aligned}
& G=\left\{(x, y) \mid x+y \geq 0,1-\frac{x}{\Delta}-\frac{y}{\Delta} \geq 0, x \leq \Delta, y \leq \Delta\right\}: \\
& \frac{\partial u}{\partial t}=\Delta u-e^{-t}\left((x+y)(x-\Delta)(y-\Delta)\left(1-\frac{x}{\Delta}-\frac{y}{\Delta}\right)\right. \\
& \left.\quad+4(x-\Delta)\left(1-\frac{x}{\Delta}-\frac{3 y}{2 \Delta}\right)+4(y-\Delta)\left(1-\frac{3 x}{2 \Delta}-\frac{y}{\Delta}\right)\right)
\end{aligned}
$$

$(x, y) \in G, t>0$

$u(x, y, 0)=(x+y)(x-\Delta)(y-\Delta)\left(1-\frac{x}{\Delta}-\frac{y}{\Delta}\right),\left.u(x, y, t)\right|_{\partial G}=0$.

Let us divide the region with coordinate axes into triangles (Figure 1):

$G=T_{1} \bigcup T_{2} \bigcup T_{3}, T_{1}=\{(x, y) \mid-\Delta \leq x \leq 0,-x \leq y \leq \Delta\}$, $T_{2}=\{(x, y) \mid x \geq 0, y \geq 0, x+y \leq \Delta\}, T_{3}=\{(x, y) \mid 0 \leq x \leq \Delta$, $-x \leq y \leq 0\}$. Denote $u(0, y, t)=\psi(y, t), u(x, 0, t)=\varphi(x, t)$.

Considering the symmetry of the problem, we will obtain $\varphi(x, t)=\psi(x, t)$.

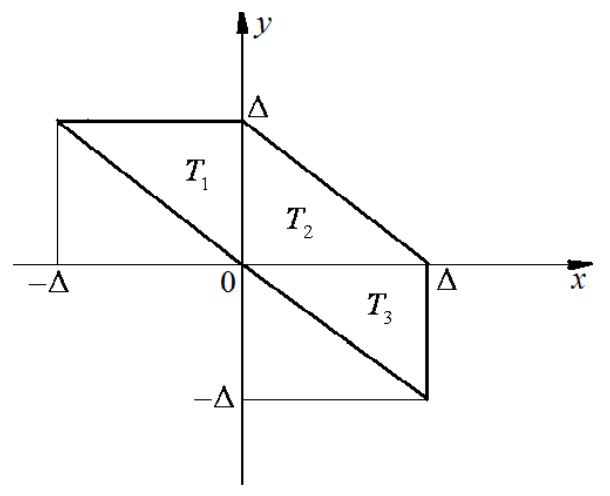

Figure 1. Partition of region $G$ using coordinate axes into triangles $T_{1}, T_{2}, T_{3}$.

Using the above statements, lets construct the function $u(x, y, t)$ :

$$
u(x, y, t)=\left\{\begin{array}{c}
\left(1+\frac{x}{\Delta}\right) \varphi(y, t)-\left(1-\frac{y}{\Delta}\right) \varphi(-x, t),-\Delta \leq x \leq 0,-x \leq y \leq \Delta, \\
\left(1+\frac{x}{\Delta}\right) \varphi(y, t)\left(1+\frac{y}{\Delta}\right) \varphi(-x, t)-\frac{x}{\Delta} \varphi(\Delta-y, t), \\
\frac{y}{\Delta} \varphi(\Delta-x, t), x \geq 0, y \geq 0, x+y \leq \Delta, \\
\left(1+\frac{x}{\Delta}\right) \varphi(x, t)-\left(1+\frac{x}{\Delta}\right) \varphi(-y, t), 0 \leq x \leq \Delta,-x \leq y \leq 0 .
\end{array}\right\}
$$

This function satisfies the homogeneous boundary conditions regardless of the choice of function $\varphi(x, t)$ and coincides with $\varphi(x, t)$ on the line $y=0$ and with the function $\varphi(y, t)$ on the line $x=0$, if $\varphi(0, t)=0, \varphi(\Delta, t)=0 \forall t \geq 0$.

We find the function $\varphi(x, t)$ in the form of first degree spline:

$\varphi(x, t)=\sum_{k=1}^{M-1} C_{k}(t) h_{\mid k}(M x-k), h(z)=\frac{1}{2}(|z-1|-2|z|+|z+1|)$.
Functions $C_{k}(t), k=\overline{1, M-1}$ we find using minimization of expression

$$
J(u)=\iint_{G}\left(\left(\frac{\partial u}{\partial x}\right)^{2}+\left(\frac{\partial u}{\partial y}\right)^{2}+2\left(\frac{\partial u}{\partial t}-f(x, y, t)\right) u\right) d x d y,
$$

using the method of local potential (according to which variation of expression $J(u)$ by function $u$ is performed so that member $u \frac{\partial u}{\partial t}$ of the Euler equation corresponds to $\left.\frac{\partial u}{\partial t}\right)$. This leads to a system of linear differential equations relatively $C_{k}(t), k=\overline{1, M-1}$ :

$$
A \frac{\partial C(t)}{\partial t}=B C(t)+F(t), t>0, \quad C(0)=C_{0}, C(t)=\left(C_{1}(t), \ldots, C_{M-1}(t)\right)
$$

Matrices $A, B, F$ are found by integration in the region $G$ of the expressions

$$
\begin{aligned}
& \frac{\partial}{\partial x} w_{i}(x, y) \cdot \frac{\partial}{\partial x} w_{j}(x, y), \quad \frac{\partial}{\partial y} w_{i}(x, y) \cdot \frac{\partial}{\partial y} w_{j}(x, y), \\
& w_{i}(x, y) w_{j}(x, y), \quad f(x, y, t) w_{\delta}(x, y)
\end{aligned}
$$

$(i, j=\overline{1, M-1})$, where considered that $u(x, y, t)=\sum_{i=1}^{M-1} C_{i}(t) w_{i}(x, y)$. Specific explicit expressions for the functions $w_{i}(x, y)$ omitted.

We perform several computational experiments for this problem, whose results are listed below. At $\Delta=0,5, M$ $=5$ we obtained the following values of the approximate solution (Table 1) and the exact solution (Table 2).

Table 1. The values of the approximate solution

\begin{tabular}{|l|c|c|c|c|}
\hline $\mathbf{t}$ & $\begin{array}{c}\mathbf{u} \\
(\mathbf{0 , 1 ; 0 ; t )}\end{array}$ & $\mathbf{u}(\mathbf{0 , 2} ; \mathbf{0} ; \mathbf{t})$ & $\mathbf{u}(\mathbf{0 , 3} \mathbf{3} \mathbf{0} \mathbf{t})$ & $\mathbf{u}(\mathbf{0 , 4 ; 0 ; t )}$ \\
\hline 0 & 0,0160 & 0,0180 & 0,0120 & 0,0040 \\
\hline 0,0001 & 0,0159 & 0,0181 & 0,0121 & 0,0041 \\
\hline 0,0002 & 0,0159 & 0,0181 & 0,0122 & 0,0041 \\
\hline 0,0003 & 0,0158 & 0,0182 & 0,0122 & 0,0042 \\
\hline 0,0004 & 0,0157 & 0,0182 & 0,0123 & 0,0043 \\
\hline 0,0005 & 0,0157 & 0,0183 & 0,0124 & 0,0043 \\
\hline 0,0006 & 0,0156 & 0,0184 & 0,0125 & 0,0044 \\
\hline 0,0007 & 0,0156 & 0,0184 & 0,0126 & 0,0045 \\
\hline 0,0008 & 0,0155 & 0,0185 & 0,0126 & 0,0046 \\
\hline 0,0009 & 0,0155 & 0,0185 & 0,0127 & 0,0046 \\
\hline 0,001 & 0,0154 & 0,0186 & 0,0128 & 0,0047 \\
\hline
\end{tabular}


Table 2. The values of the exact solution

\begin{tabular}{|l|c|c|c|c|}
\hline $\mathbf{t}$ & $\begin{array}{c}\text { ut } \\
\mathbf{( 0 , 1 ; 0 ; t )}\end{array}$ & $\begin{array}{c}\text { ut } \\
(\mathbf{0 , 2 ; 0 ; t )}\end{array}$ & ut $\mathbf{( 0 , 3 ; 0 ; t )}$ & ut $(\mathbf{0 , 4 ; 0 ; t )}$ \\
\hline 0 & 0,0160 & 0,0180 & 0,0120 & 0,0040 \\
\hline 0,0001 & 0,0160 & 0,0180 & 0,0120 & 0,0040 \\
\hline 0,0002 & 0,0160 & 0,0180 & 0,0120 & 0,0040 \\
\hline 0,0003 & 0,0160 & 0,0180 & 0,0120 & 0,0040 \\
\hline 0,0004 & 0,0160 & 0,0180 & 0,0120 & 0,0040 \\
\hline 0,0005 & 0,0160 & 0,0180 & 0,0120 & 0,0040 \\
\hline 0,0006 & 0,0160 & 0,0180 & 0,0120 & 0,0040 \\
\hline 0,0007 & 0,0160 & 0,0180 & 0,0120 & 0,0040 \\
\hline 0,0008 & 0,0160 & 0,0180 & 0,0120 & 0,0040 \\
\hline 0,0009 & 0,0160 & 0,0180 & 0,0120 & 0,0040 \\
\hline 0,001 & 0,0160 & 0,0180 & 0,0120 & 0,0040 \\
\hline
\end{tabular}

Also given the values of the approximate solution $u$ $\left(x_{i} ; y_{j} ; 0,001\right)$ (Table 3$)$ and the exact solution $u t\left(x_{i} ; y_{j}\right.$ $; 0,001)$ (Table 4$)$ in the nodes of grid $x_{i}=-\Delta+\frac{\Delta}{M}(i-1)$ , $y_{j}=-\Delta+\frac{\Delta}{M}(j-1), i=\overline{1,2 M+1}, j=\overline{1,2 M+1}$, belonging to the region $G$.

\section{Conclusion}

The paper proposes a general method for the solution of non-stationary heat conduction problem, which uses the structure of the approximate solution in the form of operator of the interlineation on a given system of lines (interlineation of functions is the restoration of the function with the help of its traces on the system of lines). The

Table 3. The values of the approximate solution $u\left(x_{i} ; y_{j} ; 0,001\right)$

\begin{tabular}{|l|l|l|l|l|l|c|c|c|c|c|c|}
\hline $\mathbf{i} \mathbf{j}$ & $\mathbf{1}$ & $\mathbf{2}$ & $\mathbf{3}$ & $\mathbf{4}$ & $\mathbf{5}$ & $\mathbf{6}$ & $\mathbf{7}$ & $\mathbf{8}$ & $\mathbf{9}$ & $\mathbf{1 0}$ & $\mathbf{1 1}$ \\
\hline 1 & & & & & & & & & & \\
\hline 2 & & & & & & & & & & \\
\hline 3 & & & & & & & & & 0 & $-0,0007$ & 0 \\
\hline 4 & & & & & & & & 0 & 0,0002 & $-0,0009$ & 0 \\
\hline 5 & & & & & & & 0 & 0,0056 & 0,0041 & 0,0007 & 0 \\
\hline 6 & & & & & & 0 & 0,0154 & 0,0186 & 0,0128 & 0,0047 & 0 \\
\hline 7 & & & & & 0 & 0,0154 & 0,0228 & 0,0197 & 0,0099 & 0 \\
\hline 8 & & & & 0 & 0,0056 & 0,0186 & 0,0197 & 0,0120 & 0 & \\
\hline 9 & & & 0 & 0,0002 & 0,0041 & 0,0128 & 0,0099 & 0 & & \\
\hline 10 & & 0 & $-0,0007$ & $-0,0009$ & 0,0007 & 0,0047 & 0 & & & \\
\hline 11 & 0 & 0 & 0 & 0 & 0 & 0 & & & & \\
\hline
\end{tabular}

Table 4. The values of the exact solution $u\left(x_{i} ; y_{j} ; 0,001\right)$

\begin{tabular}{|l|l|l|l|l|l|l|l|l|l|l|l|}
\hline $\mathbf{i} \mathbf{j}$ & $\mathbf{1}$ & $\mathbf{2}$ & $\mathbf{3}$ & $\mathbf{4}$ & $\mathbf{5}$ & $\mathbf{6}$ & $\mathbf{7}$ & $\mathbf{8}$ & $\mathbf{9}$ & $\mathbf{1 0}$ & $\mathbf{1 1}$ \\
\hline 1 & & & & & & & & & & \\
\hline 2 & & & & & & & & & & \\
\hline 3 & & & & & & & & & 0 & 0 \\
\hline 4 & & & & & & & & 0 & 0,0112 & 0,0084 & 0 \\
\hline 5 & & & & & & & 0 & 0,0144 & 0,0144 & 0,0072 & 0 \\
\hline 6 & & & & & & 0 & 0,0160 & 0,0180 & 0,0120 & 0,0040 & 0 \\
\hline 7 & & & & & 0 & 0,0160 & 0,0192 & 0,0144 & 0,0064 & 0 & 0 \\
\hline 8 & & & & 0 & 0,0144 & 0,0180 & 0,0144 & 0,0072 & 0 & \\
\hline 9 & & & 0 & 0,0112 & 0,0144 & 0,0120 & 0,0064 & 0 & & \\
\hline 10 & & 0 & 0,0064 & 0,0084 & 0,0072 & 0,0040 & 0 & & & \\
\hline 11 & 0 & 0 & 0 & 0 & 0 & 0 & & & & \\
\hline
\end{tabular}


method divides the region of consideration on rectangular or triangular elements with curvilinear, in the general case, sides, belonging to the boundary of the domain.

Given analyses allows us to draw the conclusion about effective numerical realization of proposed method for solving unsteady heat conduction problem in the case of consideration of regions of complex shape.

In the future research authors plan to generalize the proposed method for the non-stationary heat conduction problems with three space variables.

\section{References}

1. Sergienko I, Lytvyn O. The numerical implementation of the method LIDR for equation of nonstationary heat conduction. Dopovidi natsionalnoyi akademiyi nauk Ukrayiny. 1990; (10):69-73 (in Ukrainian).

2. Drobot E, Lytvyn O, Sergienko I. The numerical implementation of the method LIDR for nonstationary heat conduction equation with three spatial variables. Dopovidi natsionalnoyi akademiyi nauk Ukrayiny. 2000; (2):67-73 (in Ukrainian).

3. Lytvyn O. Computational methods. Additional sections. Kyiv, Science thought. 2005. p. 303. (in Ukrainian).

4. Lytvyn O, Lobanova L, Zaluzhna G. The numerical implementation of the method LIDR for solving unsteady heat conduction problem with two space variables. 43 Scientific Conference, Kharkiv, UIPA. 2009. p. 47. (in Ukrainian).

5. Lytvyn O, Lobanova L, Zaluzhna G. The solution of unsteady heat conduction problem for a plate by interlination finite element method. Proceedings of the International Symposium Questions of optimization of computation - XXXV. Kyiv, Institute of Cybernetics of 5. Glushkov of NAS of Ukraine. 2009; 14-9 (in Ukrainian).
6. Lytvyn O, Lobanova L, Zaluzhna G. Mathematical modeling of heat conduction with interlination. Proceedings of the XV All-Ukrainian Conference, Modern Problems of Applied Mathematics and Computer Science. - Lviv National University. 2008. p. 77. (in Ukrainian).

7. Lytvyn O, Khimich O, Yakovlev M, Lobanova L, Zaluzhna G. About one approach to solve the problem of non-stationary heat conduction with two space variables using cluster. Information and System Sciences. Procedings of Ukrainian scientific conference. Poltava: RVV PUSKU. 2010. p. 114-7. (in Ukrainian).

8. Lytvyn ON, Lobanova LS. About construction of exact solutions of boundary value problems with given class of differentiability in domains of complex shapes. Contemporary problems of mathematics, mechanics and computing sciences. Kharkov. 2011. p. 254-60.

9. Lytvyn O.N. Interlineation functions (the blending function interpolation) and some its application. International Congress Mathematics. Sect. 15: Numerical Analysis and Sci. Computing, Berlin. 1998 Aug 18-27. p. 28.

10. Lytvyn ON. Methods of a solution of boundary value problems of continuum mechanics reducing to the system of Linear (LIDE) and Nonlinear (NIDE) Integro-Differencial equations. Numerical modeling in continuum mechanics. Proceedings of the $4^{\text {th }}$ Summer Conference held in Prague. 200031 Jul - 4 Aug. p. 240-9.

11. Lytvyn $\mathrm{ON}$. Interlineation and interflation functions of many variables (blending function interpolation) and economical algorithms in the approximation theory. The International Conference of Computational Mathematics, Singapore. 2004. p. 1100-9.

12. Lytvyn ON. Interlineation and interflatation functions of many variables (blending function interpolation) and economical algorithms in the approximation theory. International Conference on Computational methods. ICCM2007. Singapore. 2004 Dec 14-15. p. 173. 